\title{
EchoGéo
}

$7 \mid 2008$

Les nouveaux enjeux régionaux dans l'océan Indien occidental

\section{Une île en mutation}

Infrastructures, aménagement et développement à La Réunion

\section{Thierry Simon}

\section{(2) OpenEdition \\ Journals}

Édition électronique

URL : https://journals.openedition.org/echogeo/8003

DOI : 10.4000/echogeo.8003

ISSN : 1963-1197

Éditeur

Pôle de recherche pour l'organisation et la diffusion de l'information géographique (CNRS UMR 8586)

\section{Référence électronique}

Thierry Simon, «Une île en mutation », EchoGéo [En ligne], 7| 2008, mis en ligne le 15 octobre 2008, consulté le 31 juillet 2021. URL : http://journals.openedition.org/echogeo/8003 ; DOI : https://doi.org/ $10.4000 /$ echogeo.8003

Ce document a été généré automatiquement le 31 juillet 2021.

EchoGéo est mis à disposition selon les termes de la licence Creative Commons Attribution - Pas d'Utilisation Commerciale - Pas de Modification 4.0 International (CC BY-NC-ND) 


\title{
Une île en mutation
}

\author{
Infrastructures, aménagement et développement à La Réunion
}

\section{Thierry Simon}

1 L'île de La Réunion semble désormais de plus en plus fréquemment présentée comme une « région européenne ultrapériphérique » de l'océan Indien : une « RUP », au même titre que sept autres espaces insulaires atlantiques et caraïbes, historiquement intégrés, de fait, à l'espace économique européen depuis 1957. Il y a peu encore l'ancienne île Bourbon apparaissait plutôt comme étant une France " australe », terre française gérée comme telle, instituée en " département 》 (1946) et en «région» (1983) : vivant ainsi une dualité administrative intéressante, inscrite dans une même enveloppe insulaire réduite $\left(2512 \mathrm{~km}^{2}\right)$.

2 Longtemps tenu à l'écart, cet espace insulaire a été fortement marqué par un souséquipement réel et par une véritable misère, comme par de très fortes inégalités socioéconomiques. Mais, en quelques décennies, l'île s'est profondément transformée. La population a plus que triplé depuis 1946, année charnière de la « départementalisation »... Et, désormais, il ne semble pas exagéré d'avancer que cette contrainte démographique, qui semblait si préoccupante et tant redoutée dans les années $1950^{1}$, a constitué un motif permanent de changement, de mutation, d'initiatives et de réalisations inscrites dans un double défi : équiper et aménager un territoire insulaire souvent très contraignant, sans faire d'erreur irréversible et majeure, dans l'urgence permanente ${ }^{2}$, avec des moyens humains et financiers obligatoirement conséquents. Ce défi est sans cesse relancé, d'une année sur l'autre, d'un programme ou d'un projet à l'autre: aucun immobilisme n'est permis, aucune pause ne peut s'envisager dans cette mutation invariable, incessante et accélérée qui transforme en profondeur la société, l'économie et les paysages insulaires.

\section{Une géographie des contraintes et des possibles}

3 Nul doute, un fait majeur mérite d'être souligné d'emblée: l'espace insulaire réunionnais impose depuis toujours des contraintes très fortes. L'île se trouve régulièrement exposée à des aléas nombreux et souvent majeurs : volcanisme éruptif, 
précipitations considérables (avec des records mondiaux...), dégâts cycloniques récurrents, instabilité des pentes et mouvements de terrain permanents,... Ici, on ne peut concevoir que de manière purement formelle, ou rêvée, une certaine langueur insulaire tropicale douce et infinie. Celle qui est perçue de l'hémisphère nord, comme étant un apanage de ces territoires lointains et océaniques, souvent mal compris, car mesurés à l'aune d'idées reçues. La réalité est toute différente : l'île est rude. Elle est l'expression d'une violence et d'une dureté intrinséques, naturelles et peut-être même indispensables, car participant à la construction d'une identité. Cette réalité influe d'ailleurs encore très fortement, et de longue date, la perception que la population a de son espace: "Île à peur", comme l'analyse Prosper Eve, (Eve, 1992), où les dangers volcaniques et cycloniques demeurent très prégnants, mais où rien ne doit faire reculer les hommes dans leurs entreprises.

La nature volcanique de sa construction fait que ce territoire est constamment exposé à des risques réels, désormais très bien mesurés et de mieux en mieux connus, donc moins redoutés qu'auparavant. Il ne faut pas forcément s'arrêter, comme c'est trop souvent le cas pour d'évidentes raisons «spectaculaires" et médiatiques, sur les épisodes éruptifs récurrents et souvent très étonnants du Piton de la Fournaise. Il figure parmi les appareils volcaniques les plus actifs au monde. Ces éruptions sont généralement contenues dans "l'enclos " (inhabité) et menacent, fort heureusement, assez rarement l'habitat et les activités rurales périphériques. Il s'agit surtout de prendre en compte les conséquences dues à l'édification même de l'île sur la très longue durée. Ce double cône émergé est une juxtaposition et une imbrication de boucliers volcaniques complexes : un feuilletage gigantesque de laves, scories, brèches et tufs très variés dans leur composition, leur épaisseur, leur fragmentation et donc dans leur résistance à l'érosion. C'est évidemment d'abord et avant tout en matière d'aménagement que ces contraintes géologiques incontournables se font très fortement ressentir. Toutes les difficultés géotechniques peuvent se trouver réunies et compliquer alors très sérieusement, voire entraver, la tâche des équipes chargées des plus grands programmes d'infrastructures de transport, comme d'habitat. 
Figure 1 - Cirque de Cilaos

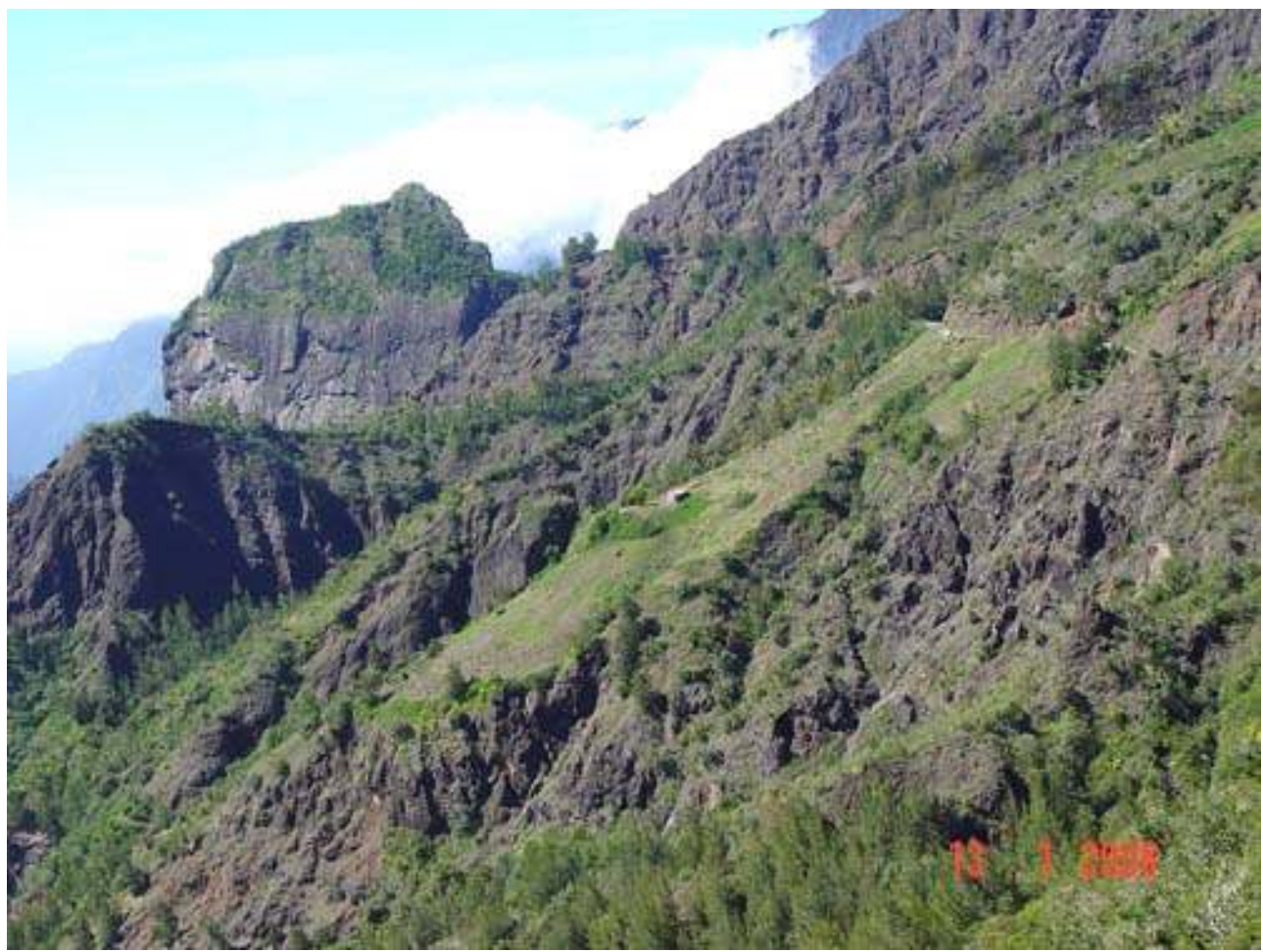

5 Les très fortes pentes n'ont pas empêché des hommes de s'installer et de mettre en culture : îlet Méon. Noter aussi, la route (D 242) reliant Cilaos à îlet à cordes, fréquemment coupée par des éboulis. Au premier plan, paysage de "bad lands", témoin de la vigueur et de l'ampleur des processus érosifs. Cliché : Th. Simon, 2008. 
Figure 2 - Les quartiers dionysiens du Moufia (premier plan) et de la Bretagne (second plan)

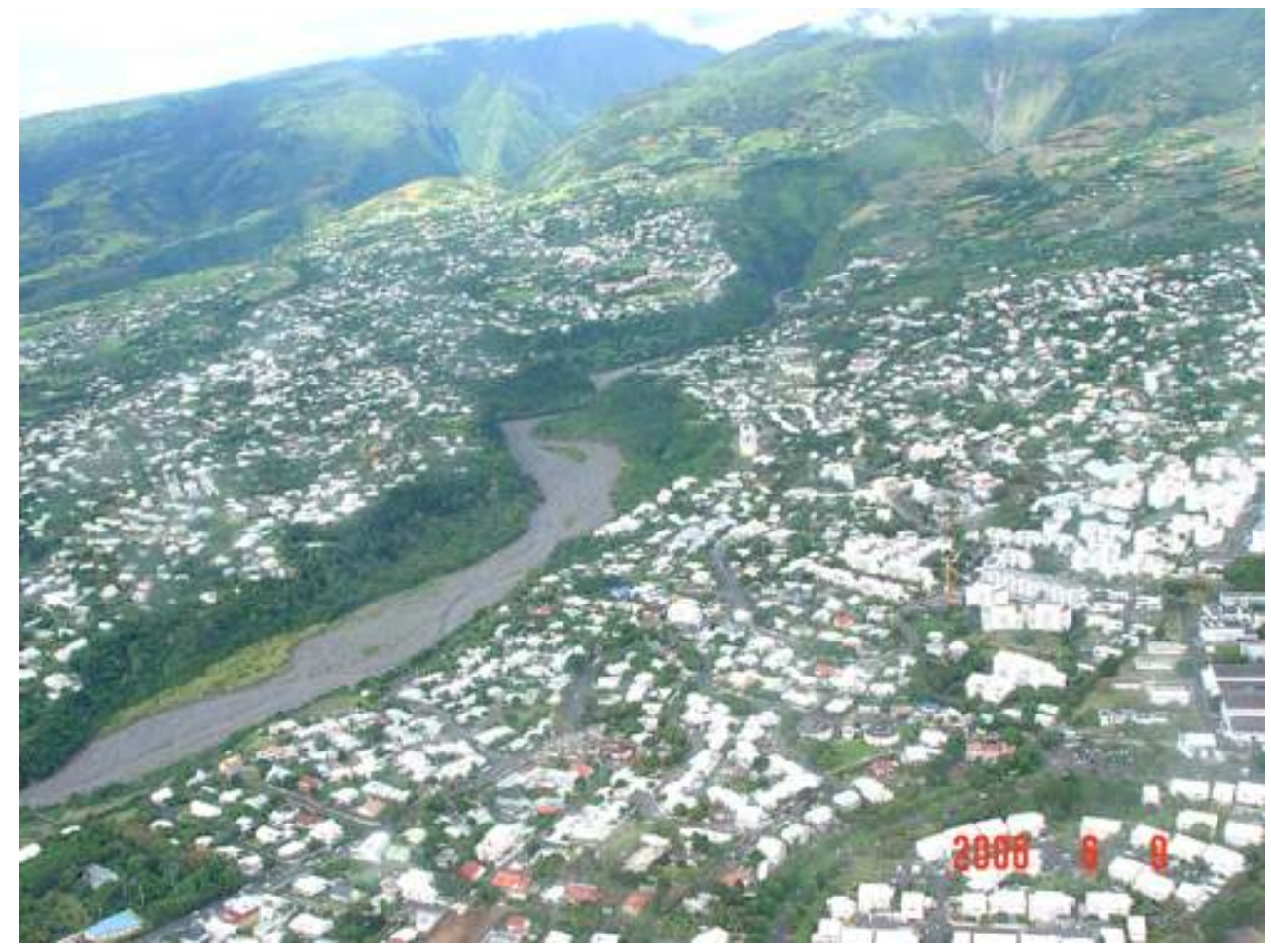

Séparés par la profonde ravine du Chaudron, dont on aperçoit, à l'arrière plan le fond de vallée et la cascade. L'habitat, constitué de petits immeubles collectifs et de "cases ", progresse graduellement vers les « hauts », en consommant une partie des espaces agricoles, voués au maraîchage et aux cultures fruitières (ananas). Les ravines constituent des obstacles difficilement franchissables et segmentent l'espace urbain. Cliché : Th. Simon, 2006.

6 Les processus géodynamiques à l'œuvre et les formes de relief induites impressionnent par leur vigueur (figure 1). Surtout, formes et processus ne vont certainement pas faciliter les programmes d'aménagement, quelle que soit leur ampleur ou leur nature. Le réseau radial et très dense des ravines et des grandes vallées découpe les planèzes et constitue une multitude d'obstacles à franchir. Au cœur de l'île, les cirques et les grandes vallées encaissées sont autant d'espaces difficilement parcourus, habités, reliés et mis en valeur. Ces «écarts » ont d'ailleurs été tardivement occupés, sont demeurés longtemps isolés, le restent souvent encore. Nombre « d'îlets » constituent en quelque sorte des archipels dans le cœur même de l'île. Même s'ils accueillent une population limitée et s'ils restent encore largement en marge des dynamiques économiques nouvelles ${ }^{3}$, ces îlets demeurent représentatifs d'une volonté sans cesse réaffirmée: même si les risques existent, même si les difficultés paraissent insurmontables, l'essentiel de l'espace insulaire doit être parcouru et peut être occupé, même si on considère que $40 \%$ de l'espace est en réalité utilisable, (Jauze, 2003). Dans un contexte de croissance démographique soutenue, d'urbanisation et de périurbanisation accélérées, (Ninon, 1995 ; Jauze \& Ninon, 1999 ; Thinon et al., 2007), l'espace insulaire demeure toujours très fortement contraignant et les questions foncières revêtent évidemment un caractère stratégique pour les nombreux programmes, en cours ou envisagés, d'infrastructures et d'habitat. La progression générale et continue de l'habitat des basses pentes des planèzes vers les "hauts" de l'île (figure 2) est probablement révélatrice d'une dynamique spatiale trop longtemps cantonnée dans une dichotomie qui peut sembler désormais partiellement remise en cause. Même si 
une "distribution contrastée » de la population pouvait à juste titre encore être avancée récemment, (Lajoie, 2003), à l'échelle du territoire insulaire tout entier, les extensions urbaines des dernières années paraissent en mesure de modifier la donne. De fait, "Aujourd'hui, ce sont les premières pentes qui supportent l'essentiel de la croissance urbaine ", (Ninon, 2003) et cette dynamique va nettement en s'amplifiant, relayée aussi par un évident et préoccupant mitage, très inégalement maîtrisé, de la sole cannière aux périphéries des plus grands centre urbains (figure 3).

7 Il semble donc que le processus inévitable de croissance urbaine ne soit pas entièrement « sous contrôle » et qu'il y ait là l'un des enjeux majeurs auxquels l'île soit maintenant confrontée. De multiples travaux de prospective territoriale commencent à paraître, (parmi les plus récents: Thinon et al., 2007; Lajoie \& Hagen-Zanker, 2007). Mais, ils ne contribuent pas toujours ni à clarifier les enjeux, ni à mobiliser vraiment ceux qui ont le pouvoir de décider: ils peuvent même, a contrario, leur servir d'argument pour ne rien décider, tant les scénarios sont dissemblables. En effet, l'inquiétude réelle réside plus dans la capacité et la volonté des «encadrements » à mettre en œuvre des schémas validés ou des décisions approuvées. Il existe ainsi un arsenal réglementaire impressionnant: un Schéma d'Aménagement Régional (SAR), déjà ancien (1995) et en cours de révision, des Plans Locaux d'Urbanisme souvent peu consensuels, contestés ou en "révision" permanente, les Plans de Prévention des Risques (PPR) qui demeurent largement lettre morte, des zonages multiples et diverses opérations de rénovation urbaine... Cette multitude de dispositifs et de schémas emboités est, dans les faits, assez largement illisible, voire franchement incompréhensible. Elle complexifie d'une certaine manière, peut-être à dessein parfois, les entreprises d'aménagement et de développement local, parfois dans des contradictions réglementaires difficilement surmontables.

Des difficultés importantes subsistent très concrètement en matière d'aménagement du territoire, malgré les divers outils mobilisés, en dépit aussi des intentions affichées et réitérées régulièrement. En juin 2006, deux ans après que le principe d'une révision du SAR a été adopté, la Préfecture de Région, s'adressant à l'exécutif régional, révèle l'ampleur des difficultés engendrées par cette complexité liée au grand nombre d'acteurs et de réglementations. "Cette révision est l'occasion d'une nécessaire réflexion stratégique qualifiant l'intérêt régional, ainsi que d'une opportunité de convergence des différentes politiques publiques $»^{4}$. En creux, peut-on comprendre que ce serait bien l'inverse qui jusqu'alors aurait prévalu ? À savoir, une absence de vision stratégique et une juxtaposition incohérente et néfaste d'intérêts trop variés pour que les vrais enjeux liés à la cohérence territoriale puissent être compris. Ainsi, par exemple, il est assez surprenant de constater qu'au moment où la phase cruciale de révision concertée et collective du Schéma d'Aménagement Régional (SAR) est engagée, le Conseil Général décide de publier un Schéma Départemental d'Aménagement et de Développement Durable (SDADD) intitulé "Construisons La Réunion des hommes et des territoires ", (Conseil Général, 2007). 
Figure 3 - La région littorale de Saint-André, à Champ Borne, vue en direction du nord-ouest

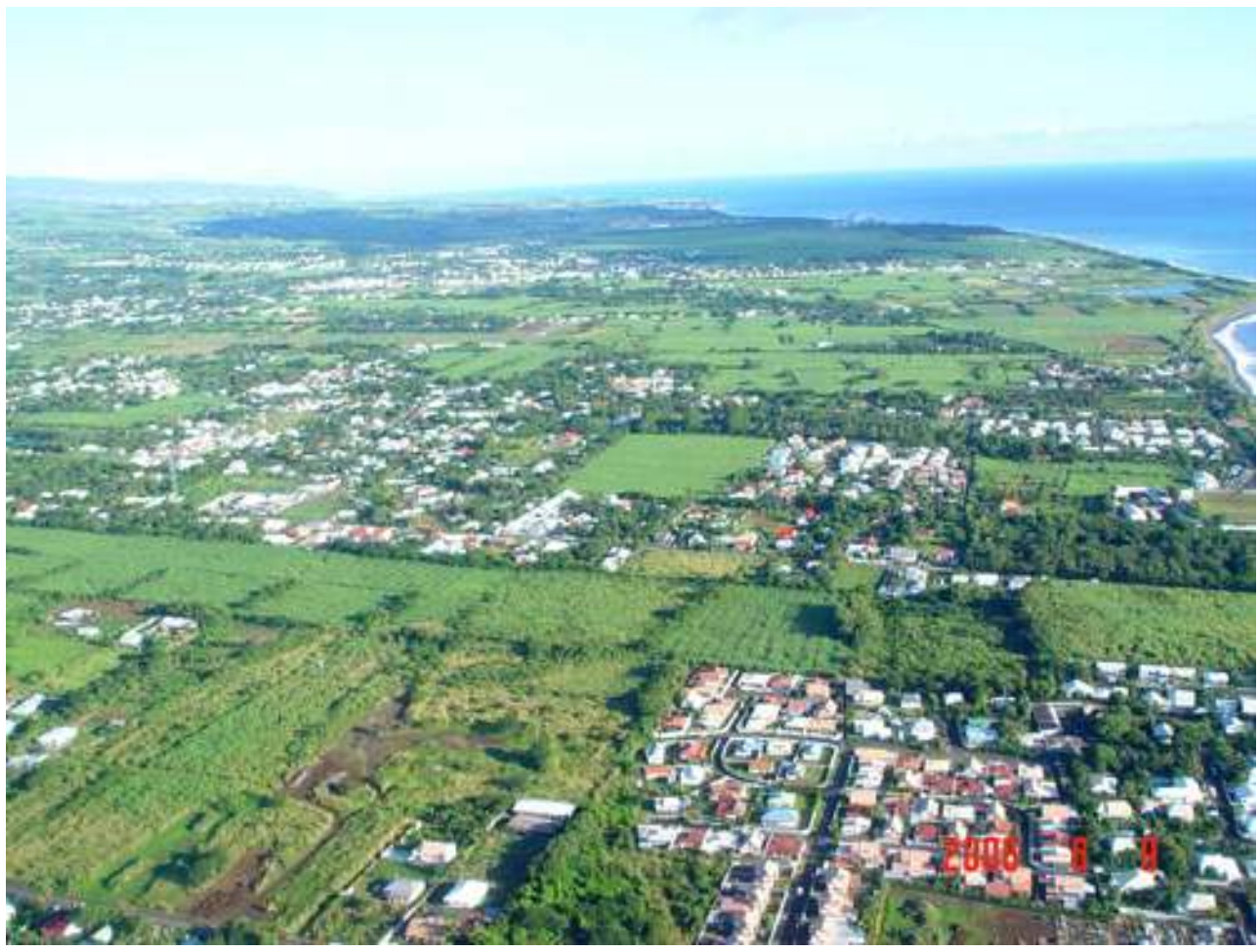

La sole cannière de cette partie du « Bon pays » est en très grande partie occupée désormais par des lotissements. Au tout dernier plan, à gauche, se distingue le massif de la Montagne. Cliché:

Th. Simon, 2006

Il semble bien que ce soit ce niveau général et consensuel de réflexion stratégique sur l'aménagement qui fasse défaut et, qu'en définitive, la mise en place des grandes infrastructures ne réponde pas aux questions fondamentales et aux enjeux réels de développement, dans la durée. Sans pour autant sombrer dans une provocation excessive, ne peut-on envisager que les "grands travaux » ne seraient en définitive qu'un pis-aller, un coûteux mais très spectaculaire et « médiatisable » écran de fumée, masquant l'absence ou la faiblesse d'une réflexion prospective et stratégique sur le long terme. Ainsi, la gestion conjointe, raisonnée et harmonieuse, de l'extension urbaine et des espaces naturels ou agricoles est trop rarement abordée, car elle soulève des questions spéculatives très (trop ?) délicates à gérer.

De même, une vraie politique, claire et affichée, conduite dans la continuité et sur le moyen terme, en matière d'habitat, notamment social, continue à faire défaut «alors que la part des logements insalubres reste inacceptable en dépit des fonds massifs qui y sont engagés et que l'évolution des prix du foncier engendre de nouvelles ségrégations $»^{5}$. Enfin, la prise en compte spatiale et dynamique de la dimension des risques paraît encore très largement sous-estimée et ne constitue jamais, ou très rarement, une priorité pour les décideurs et les aménageurs. Sur l'ensemble de ces problèmes, les constats sont établis depuis longtemps, les diagnostics ont été réitérés maintes fois, les schémas et programmes successifs ont été discutés et validés, mais leur mise en œuvre, surtout dans ses aspects les plus contraignants, n'est pas toujours au rendez-vous. 


\section{De projets titanesques en chantiers prométhéens}

11 On constate, par contre, que dans le domaine des grands travaux d'infrastructures, La Réunion se trouve résolument engagée dans divers programmes très ambitieux. Ils ont comme principal mérite immédiat de "tirer » une grande partie de l'activité et de générer une croissance dynamique. Toutefois, cette croissance ${ }^{6}$ est fondamentalement fragile, car très dépendante de transferts financiers massifs, tant de la part de l'Etat que de l'Union européenne. Exogène, cette croissance est donc structurellement incertaine, à long terme.

12 La question des déplacements intra-insulaires a toujours constitué un sujet central de préoccupation pour les autorités successives en charge de l'île : une sorte de rocher de Sisyphe qui n'a cessé de devenir plus imposant au fil des décennies. C'est évidemment d'abord la liaison vitale entre Saint-Paul/Le Port et Saint-Denis qui, depuis trois siècles et demi, se trouve au cœur de ces problématiques de déplacements. Le problème a commencé à revêtir une importance stratégique dès lors que la ville de Saint-Denis s'est trouvée érigée en «chef-lieu» (1669). La question des liaisons entre les espaces portuaires (rade de Saint-Paul, puis Le Port) de l'ouest de l'île et le pôle urbain principal au Nord, centre administratif et décisionnel, resurgit depuis lors continûment, sans qu'une solution durable et satisfaisante, (i.e: adaptée aux moyens de transport et aux flux) ait finalement été trouvée. Le massif de La Montagne demeure un obstacle intangible. C'est donc, en vérité, une course permanente qui est engagée et qui semble parfois vaine, car elle est depuis toujours conduite dans un compromis à trouver entre les moyens considérables à mobiliser pour franchir ce massif, le temps des décisions et celui de travaux, les mutations économiques et technologiques.

13 Aucun décideur n'est jamais resté inerte face à ce considérable défi: il s'agit là une constante qui mérite d'être soulignée. Cette constance pluriséculaire témoigne en effet d'une volonté permanente et invariable de s'affranchir des difficultés liées à l'aménagement: tout obstacle doit pouvoir être surmonté dès lors que le développement même de l'île se trouve engagé. Très tôt, des idées sont émises et des décisions sont prises afin de sécuriser les déplacements entre le Nord et l'Ouest. Un méchant chemin "cavalier ", est établi en pied de falaise, mais il cause, dès 1670, des soucis tels, qu'un tunnel pour franchir le Cap Bernard est déjà envisagé 7 . Entre 1730 et 1767, un chemin est difficilement aménagé en rebord du massif. Il s'agit de la première vraie « route » de l'île, soigneusement pavée, permettant de franchir de manière sûre (mais longue: plus de 2 heures) cette montagne problématique, demeurant toutefois impraticable aux charrois et donc aux transports de marchandises. Ce chemin existe toujours (figure 4) et constitue un élément patrimonial d'un intérêt certain.

14 L'accélération et l'amplification des échanges va conduire à une successions d'infrastructures variées : au cœur du XIX siècle sucrier, une route « de la Montagne » est tracée ${ }^{8}$, assurant un franchissement sûr aux hommes comme aux marchandises. L'option du lien « littoral » n'est pas abandonnée: en 1876, un étroit chemin littoral (" chemin Laugier») est achevé, tandis qu'en 1882, une "révolution technologique ", celle du chemin de fer, permet de relier Saint-Denis à La Possession, à travers la Montagne, par trois tunnels successifs. On a donc alors en place toutes les options de franchissement, réalisées avec opiniâtreté, ayant mobilisé aussi des moyens conséquents : la problématique d'un lien durable et «multimodal » est donc déjà non seulement posée, mais encore résolue, de manière à satisfaire aux besoins de la période. 


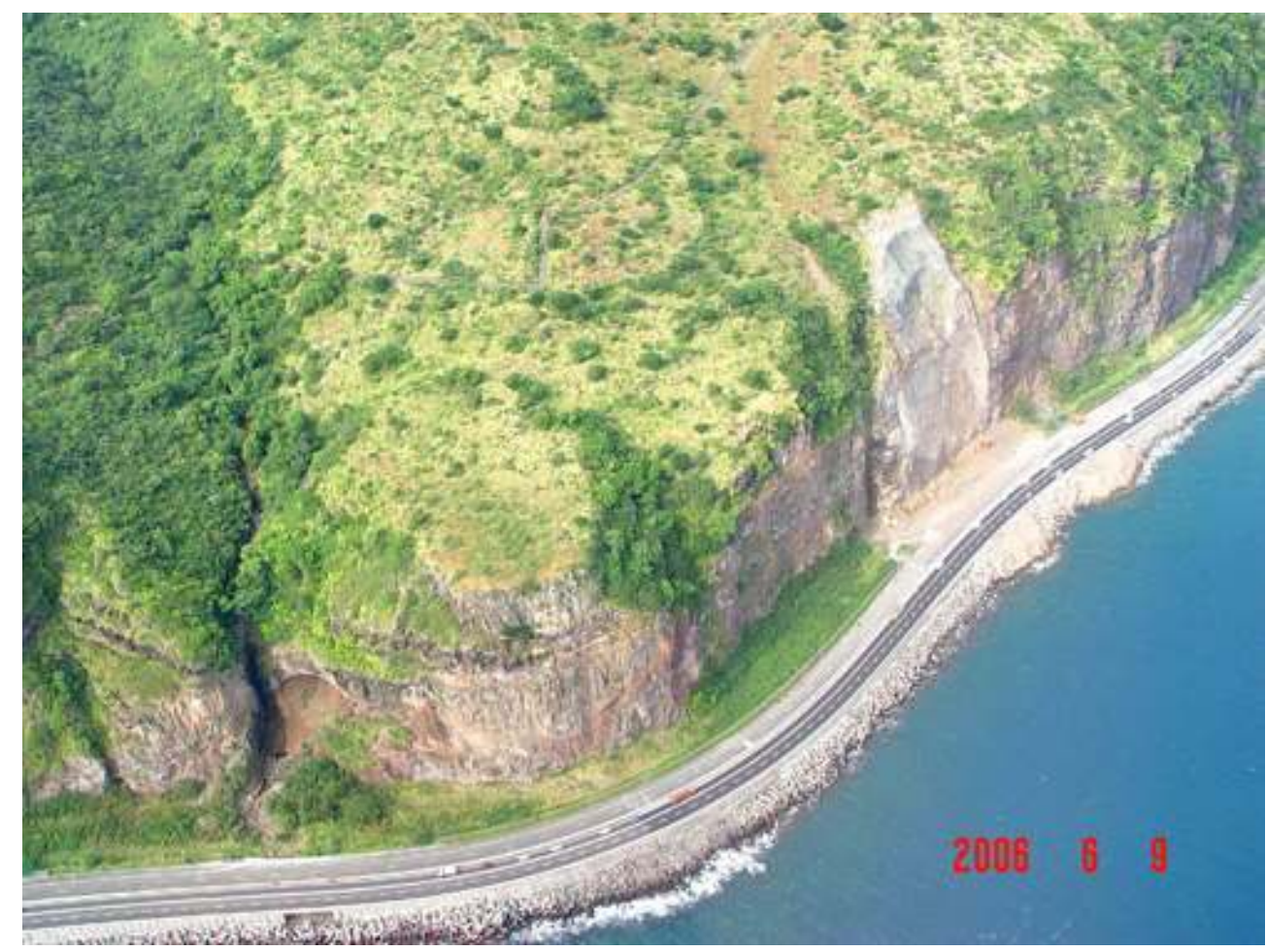

Cette infrastructure routière (2×2, en mode «normal ») est protégée, côté océan, par des tétrapodes. Côté «montagne », le danger d'éboulis est toujours présent, malgré des travaux de protection (flets, gabions). On observe ici la zone de l'éboulement majeur (effondrement d'un pan entier de falaise) du 24 mars 2006 (deux victimes). Noter aussi, sur le replat, le chemin établi au XVIIe siècle : chemin "Crémont » ou « sentier des Anglais ». Également, en contrebas immédiat du rebord de falaise, on peut distinguer (partie gauche de l'image) des traces du chemin Laugier. Cliché : Th. Simon, 2006.

15 L'arrêt de l'exploitation du chemin de fer et, surtout, la croissance conjointe du trafic routier vont, dès le début des années 60 , engendrer des difficultés majeures et croissantes. La mise en service de la coûteuse et incertaine route du littoral, de 1973 à 1976, (figure 4) n'y change rien: d'une certaine manière, le piège redoutable s'est à nouveau refermé sur l'île... Car, en effet, la donne change à grande vitesse : 280000 véhicules sur l'île en 2004, 500000 attendus (mais certainement pas espérés) vers 2020... 3500 véhicules par jour sur cette route du littoral en 1964 et... 50000 par jour en 2004. La mobilité générale des Réunionnais s'est fortement accrue. Dès lors, les études comme les promesses se succèdent pour tenter de faire face à la menace réelle d'un engorgement répété, d'une ankylose ou d'une paralysie handicapante qui viendraient certainement compromettre tous les efforts de développement. Mais, c'est bien ce choix d'un développement des transports automobiles individuels qui pose fondamentalement problème : ce choix est admis et n'est jamais clairement remis en cause. Serait-ce d'ailleurs envisageable, tant l'automobile occupe désormais une place essentielle, voire centrale, dans le quotidien économique, social et culturel de l'île?

16 Le malaise lié aux conséquences de ce choix est toutefois sous-jacent dans la décision qui a été prise de mettre récemment en œuvre un lien «tram-train »: réémergence en quelque sorte, sous une forme " hypertechnologique », du «ti train lontan » abandonné il y a quarante ans. Mais, conjointement, la « requalification » du lien routier littoral est également engagée, une nouvelle voie (une partie souterraine et une partie sur digue) 
devant être construite. Les deux projets constituent évidemment des investissements tout à fait considérables et des enjeux technologiques et techniques majeurs. Les deux chantiers à venir ont d'ailleurs nécessité un accord entre l'Etat et la Région (« accord de Matignon » en janvier 2007) portant sur un financement partagé des infrastructures. Le coût prévisionnel du «tram-train » est établi à 1250 millions d' $^{9}$, celui de la nouvelle route du littoral se situe actuellement à 900 millions d'€ ${ }^{10}$. On mesure pleinement l'ampleur des investissements à réaliser. Ces investissements prévisionnels cumulés, de l'ordre de 2300 millions d' $€$, équivalent, par comparaison, à environ 6 années de PIB des Comores (RFIC), 3 années de PIB des Seychelles, à la moitié du PIB annuel de Madagascar ${ }^{11}$.

Figure 5 - Les modalités successives de franchissement du massif de La Montagne

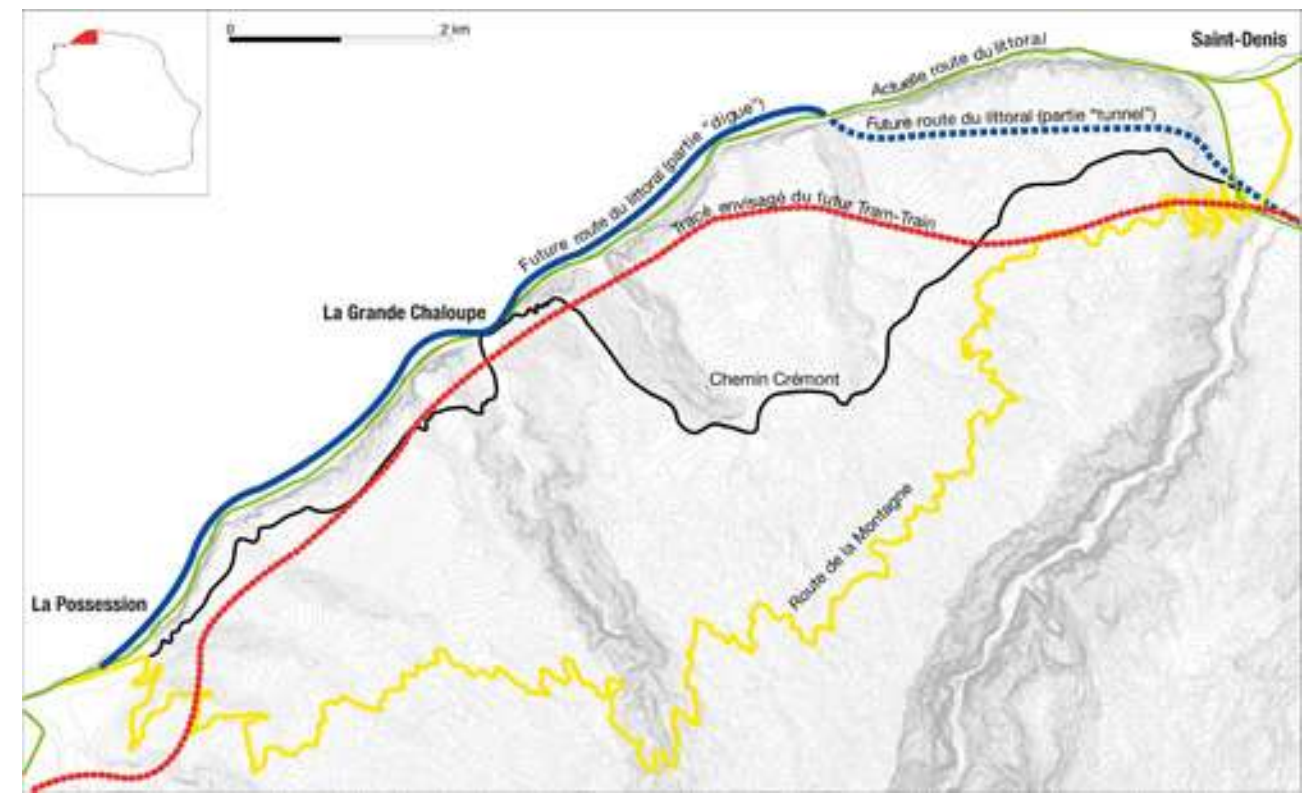

Le coût de ces deux projets gigantesques apparaît d'autant plus lourd que d'autres très grands aménagements sont encore en cours et en voie d'achèvement: la "route des tamarins " et le " basculement des eaux », chantiers très ambitieux, tous deux situés à l'ouest de l'île (figure 6). La mise en place de la "route des tamarins » a été décidée en 1998. Cette infrastructure routière moderne (2x2 voies) et nouvelle s'inscrit en réalité, elle aussi, dans une certaine continuité « historique ». En effet, par sa conception et son objet, elle semble en quelque sorte faire écho au projet, conduit à bien au XIX siècle, par le Gouverneur «bâtisseur » que fut Hubert Delisle, d'une route dans les « hauts » de l'Ouest, reliant Le Guillaume à Trois-Bassins, sur les courbes de niveau 800-900 m. Cette route Hubert Delisle constituait un vrai projet d'aménagement : elle visait à fixer, sur les pentes extérieures sous le vent, une population rurale pionnière, sur un espace rural alors mixte (canne et géranium), à structurer une nouvelle «ligne de peuplement », parfaitement dessinée depuis lors. La "route des tamarins » ne seraitelle pas en quelque sorte son « pendant » contemporain? 
Figure 6 - Le viaduc de Fleurimont, dans les « hauts » de Saint-Gilles

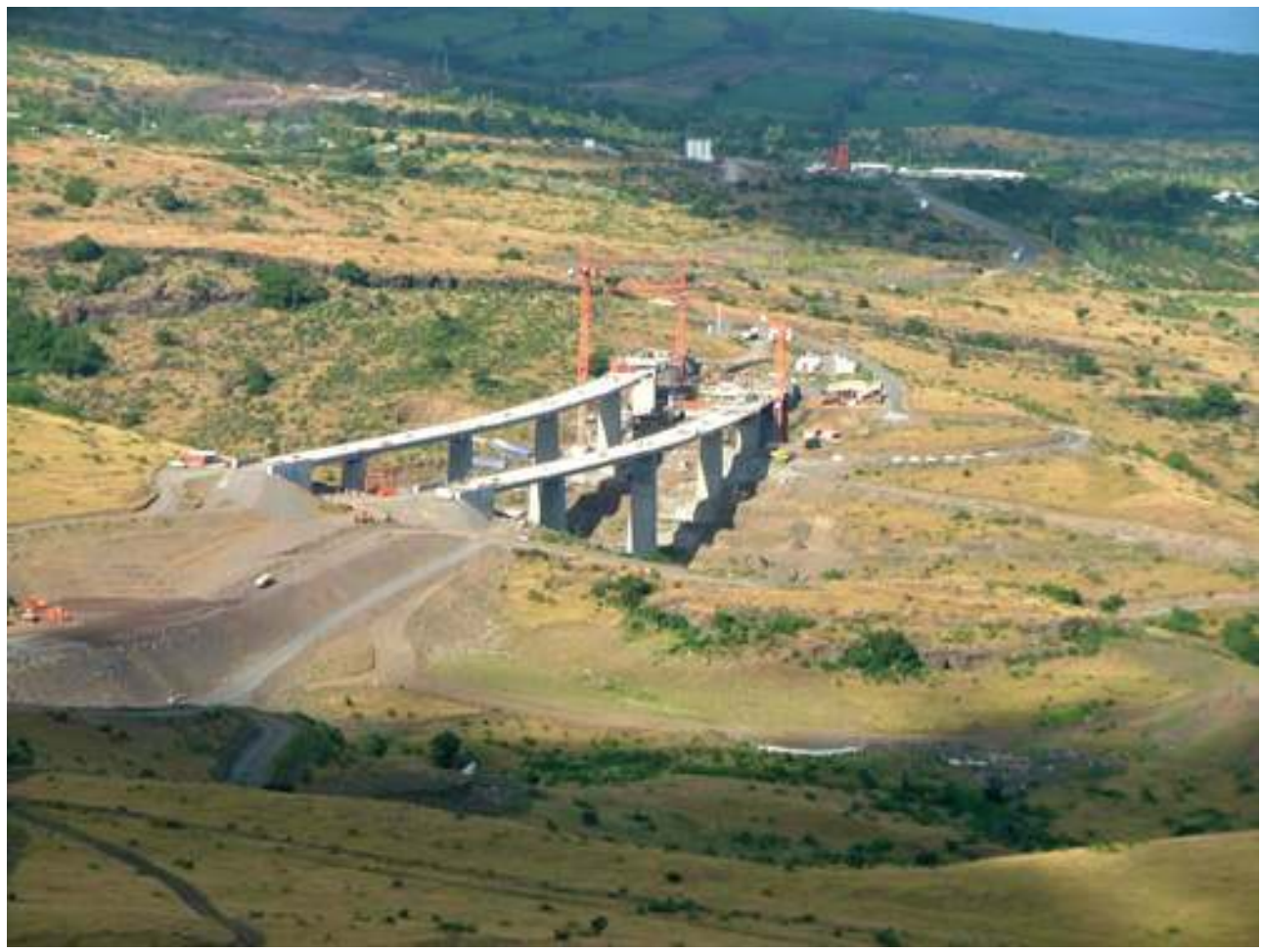

Ce viaduc est l'un des 120 ouvrages de franchissement de la route des tamarins, en juin 2006. Noter aussi que ce chantier contribue à remodeler d'ores et déjà les paysages de mi-pente, à l'ouest de l'île: routes de desserte des chantiers les plus importants, remblais et déblais considérables,... Cliché : M.A. Lamy-Giner, 2006.

18 Cette infrastructure va prochainement (2009) relier Saint-Paul à l'Etang-Salé, sur 33 kilomètres situés à mi-pente. Les propos du Président du Conseil Régional, tenus en 2006 à propos du projet, ne semblent-ils pas rentrer en résonance parfaite avec les intentions d'Hubert Delisle: «Le choix de faire passer cette route, non pas en bordure de littoral, mais en moyenne altitude, répond à une volonté politique forte : celle d'un aménagement cohérent du territoire. Cette route va permettre l'aménagement des territoires situés à mihauteur, indispensables pour accueillir une partie des 300000 habitants supplémentaires que La Réunion accueillera dans moins de 25 ans.", (Conseil Régional, 2006, p. 15). Surtout, la route est voulue pour "fluidifier » le trafic routier, sans cesse plus dense, dans cette partie balnéaire et touristique de l'espace insulaire. Il s'agirait donc d'un aménagement "structurant", à tel point que certains, certainement séduits par l'ampleur du chantier, plus que par ses effets structurants encore incertains, évoquent maintenant ouvertement un projet en tous points comparables dans les « hauts » de l'Est. Il faut convenir que la mesure de l'ouvrage (pas moins de 120 "ouvrages d'art » - certains faisant appel à des techniques entièrement nouvelles - sur $33 \mathrm{~km}$ ) est à la dimension des ambitions affichées.

19 Le chantier de "basculement des eaux » est, lui aussi, le témoin d'une autre grande ambition: il semble en réalité pratiquement d'ordre prométhéen et titanesque tout à la fois. Néanmoins, il est nettement moins spectaculaire cette fois que les infrastructures routières...Les multiples difficultés rencontrées depuis le début de cet aménagement (1989), les retards multiples et les surcoûts engendrés, ne font plus de ce projet, celui « du siècle » comme il avait été initialement baptisé imprudemment. Avec ce chantier, 
il s'agit en effet de procéder à un transfert d'Est en Ouest de réserves d'eaux, dont la ressource est jugée suffisamment abondante «au vent » et dans les cirques (Salazie et Mafate) pour qu'elle puisse bénéficier aux espaces en déficit hydrique de la côte occidentale, "sous le vent». L'idée est évidemment plus simple à énoncer que le chantier à conduire à terme. Mais il y a bien là aussi une volonté d'aménagement du territoire: "l'affichage" est orienté en direction du monde rural (développer les superficies irriguées). On peut aussi penser qu'une bonne partie de la précieuse ressource sera finalement dévolue à l'alimentation des nouveaux périmètres résidentiels de l'Ouest, d'autant que l'avenir de la production cannière est très incertain. C'est en fait un périmètre total de 7150 ha de terres cultivables (5 250 ha entre les cotes 0 et $660 \mathrm{~m}$ et 1400 ha entre les cotes 660 et $800 \mathrm{~m}$ ), intéressant plus de 3000 exploitations agricoles, qui est concerné par ce projet. Mais, ce projet doit aussi fournir un complément de ressource en eau à cinq communes importantes de l'Ouest, régulièrement en déficit : Le Port, La Possession, Saint-Paul, Trois-Bassins et Saint-Leu, c'est-à-dire à 180000 habitants, soit la quart de la population insulaire. Enfin, ce projet doit contribuer également à la recharge de la nappe de la Rivière des Galets, stratégique car elle alimente les zones industrielles portuaires.

Figure 7 - Les grands chantiers à l'ouest de lîle

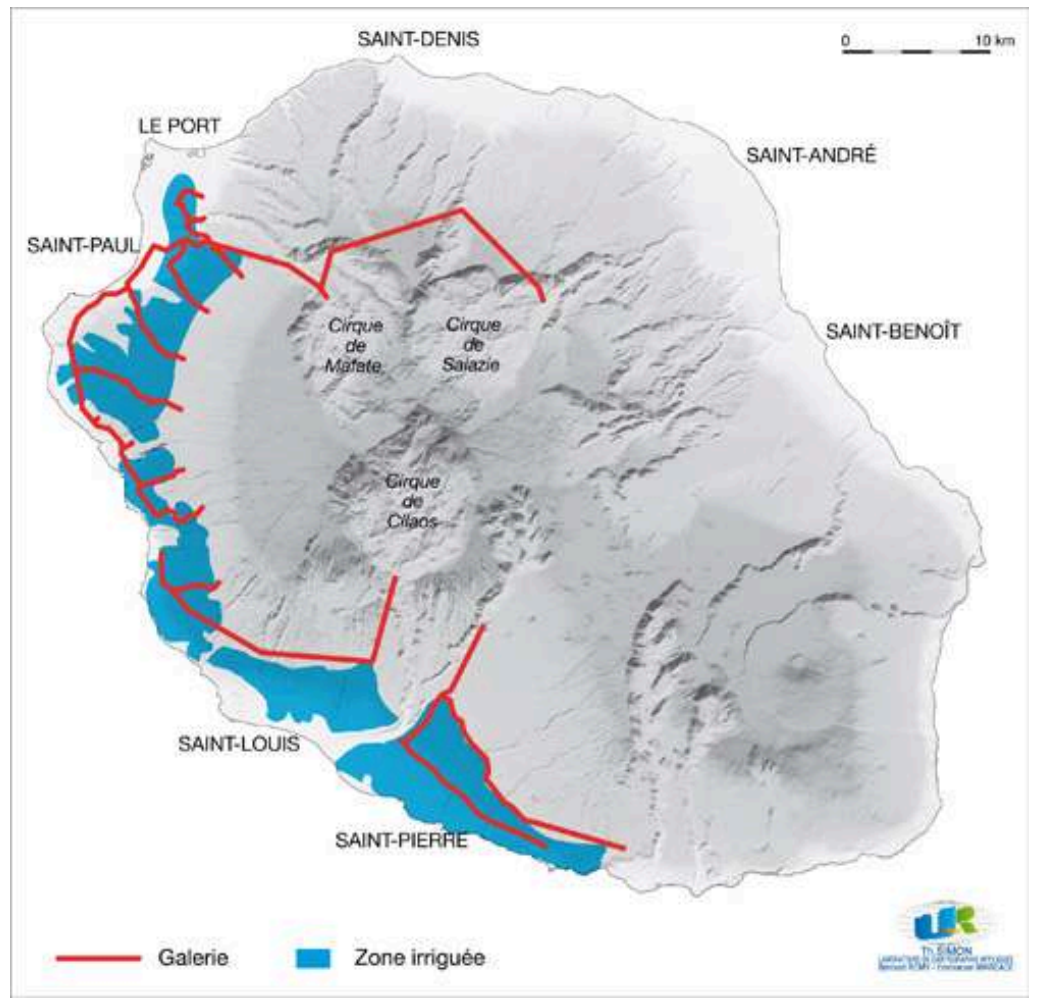




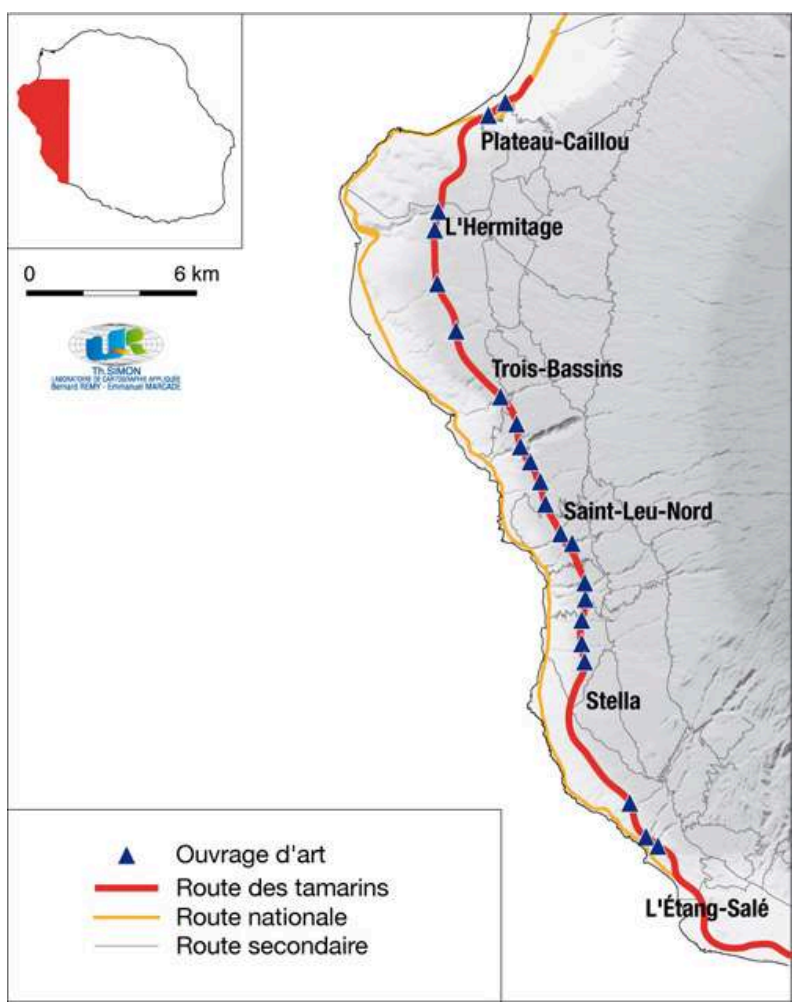

Ces grands projets traduisent clairement une part essentielle de la mutation entamée à La Réunion. À bien des égards, elle n'est plus cette "île à sucre", passée "de la colonisation à la mondialisation» (Crusol, 2007), ayant développé une société et une économie rurales et «cannières » fondamentales, même si un attachement identitaire compréhensible s'exprime encore à l'égard de ce mode de production et de vie. L'île a opéré une véritable mutation tous azimuts. Elle s'est désormais attachée à un autre « modèle » de développement, ambitieux et fragile, très complexe à gérer et à mettre en œuvre. Ce modèle commence à s'inscrire très fortement dans l'espace et les paysages (Simon, 2007). Il s'agit en effet désormais de gérer au mieux le développement urbain, de réussir de grands programmes d'équipement territoriaux (infrastructure de transport, mais aussi habitat ${ }^{12}$ ), satisfaisant au mieux aux besoins et les anticipant, créateurs de richesse et d'emplois, respectueux enfin des atouts patrimoniaux exceptionnels (naturels comme culturels) de l'île.

\section{Dégager de nouvelles perspectives « durables » ?}

L'aménagement de l'espace et le développement insulaires ne se résument évidemment pas aux grands travaux d'infrastructures, aussi spectaculaires et essentiels soient-ils, souvent très attendus aussi, même s'ils contribuent très largement à développer les activités ${ }^{13}$, ainsi qu'à remodeler les paysages. En outre, il faut bien envisager, à moyen terme, surtout dans un contexte de plus en plus restrictif sur le plan budgétaire, d'autres solutions complémentaires et d'autres perspectives de développement. Et, sur ce plan, La Réunion commence à s'engager résolument et de manière prometteuse dans deux directions qui suscitent un intérêt certain et dessinent de nouvelles pistes pour les années à venir. 
Si les travaux d'infrastructures et les réalisations du BTP en général constituent des moteurs pour la croissance, on observe aussi une nouvelle dynamique territoriale, plus diffuse mais réelle, grâce à la mise en place de très nombreuses zones d'activités, innovantes souvent, bénéficiant toujours de l'appui des collectivités locales et des structures intercommunales. Ces structures se multiplient. Elles contribuent efficacement à organiser un nouveau tissu économique désormais essentiel : 54 zones sont actuellement opérationnelles et couvrent 800 ha au total. Il est envisagé de consacrer 500 ha à ces zones d'activités d'ici 2020. La dispersion territoriale (figure 8) de ces dispositifs constitue un atout, car les déplacements induits peuvent, de la sorte, se trouver limités.

Figure 8 - Les zones d'activités

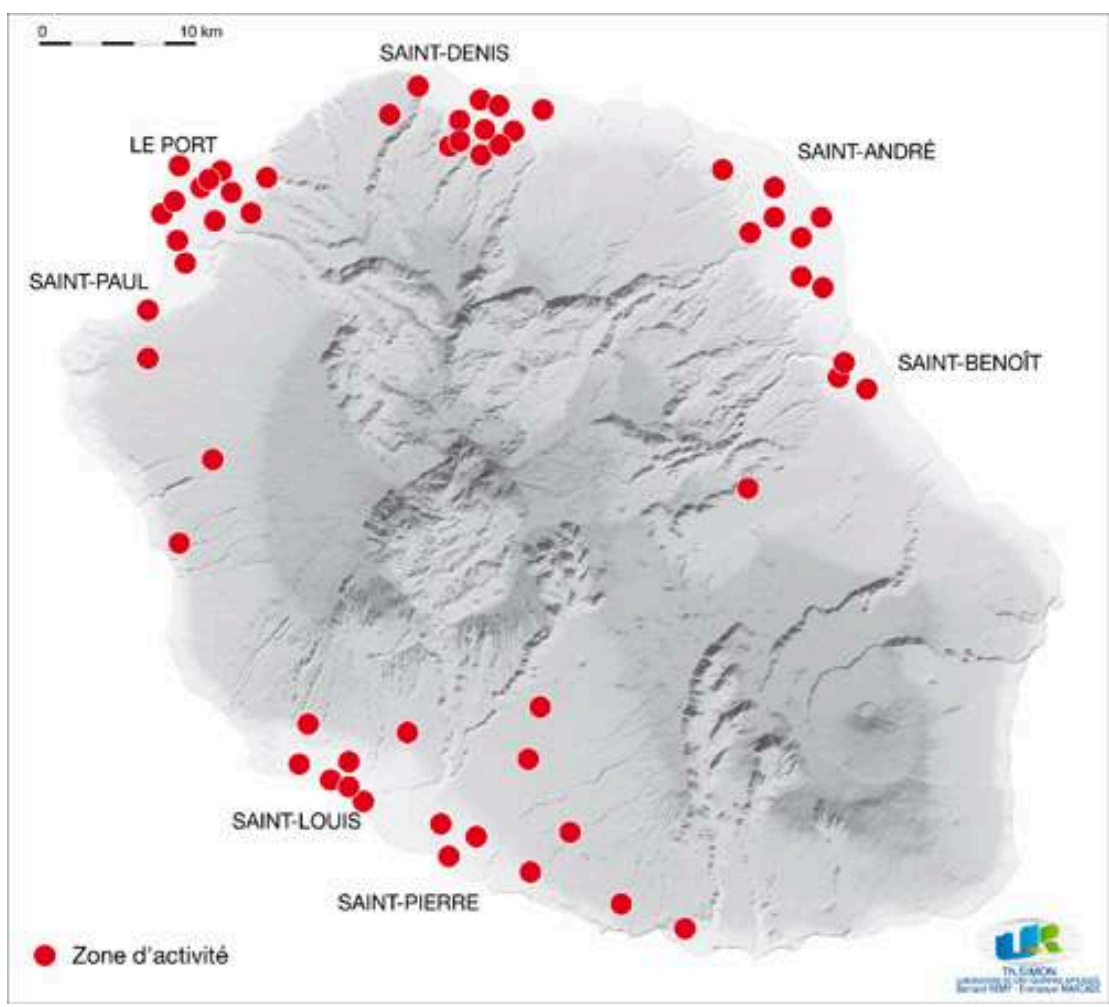

23 Mais, dans une certaine mesure, on commence aussi à s'orienter vers une graduelle spécialisation de ces zones dans certains secteurs d'activité : logistique, distribution et transport (Le Port et la Possession), agroalimentaire (Saint-Pierre), services et NTIC (Saint-Denis)... On s'achemine donc vers la création de "pôles économiques ", avec des effets de regroupement, de synergie, de cohérence et de complémentarité. Trois pôles apparaissent particulièrement dynamiques, si l'on examine en particulier les nouveaux emplois créés en une dizaine d'années (1995-2005) : Saint-Paul/Le Port/La Possession, avec 11000 nouveaux emplois créés dans ces zones, Saint-Pierre/L'Etang-Salé, avec 8500 nouveaux emplois et Saint-Denis/Sainte-Marie, avec 7800 nouveaux emplois. Plus de 2000 entreprises ont été crées grâce à ces zones et plus de $92 \%$ d'entre elles semblent durablement installées: leur "durée de vie » dépassant maintenant une décennie, elles contribuent fondamentalement désormais à un vrai dynamisme économique endogène, même quand leur activité est liée aux échanges, à la consommation et aux services, plus qu'à la production artisanale ou industrielle. 


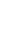

Loin de se cantonner aux seuls aspects, certes stratégiques, liés à la conservation et à la valorisation des milieux naturels et de la biodiversité (dans un contexte particulier de relatif endémisme insulaire), ce projet a su très habilement insister sur les opportunités de développement local qui doivent constituer un volet central du projet. Il est ainsi établi, par exemple, que le Parc National a vocation à participer au développement d'une urbanisation maîtrisée dans les hauts de l'île. Ce volet a longuement fait débat, car il a souvent été perçu potentiellement comme un outil supplémentaire de contrainte, par des édiles très soucieux de préserver leur capacité à gérer le foncier. Le projet doit aussi viser à favoriser la réalisation d'équipements intégrés, inciter et participer au développement d'une «économie de services» qualifiés, d'une agriculture et d'une «filière bois» de qualité. Dans le domaine du développement touristique, le Parc National doit aussi évidemment jour un rôle essentiel : mise en valeur de sites, écotourisme sur un vaste territoire, unique au monde, et qui sera peutêtre intégré prochainement au réseau des sites du Patrimoine Mondial de l'UNESCO. 
Figure 9 - Le Parc National de La Réunion

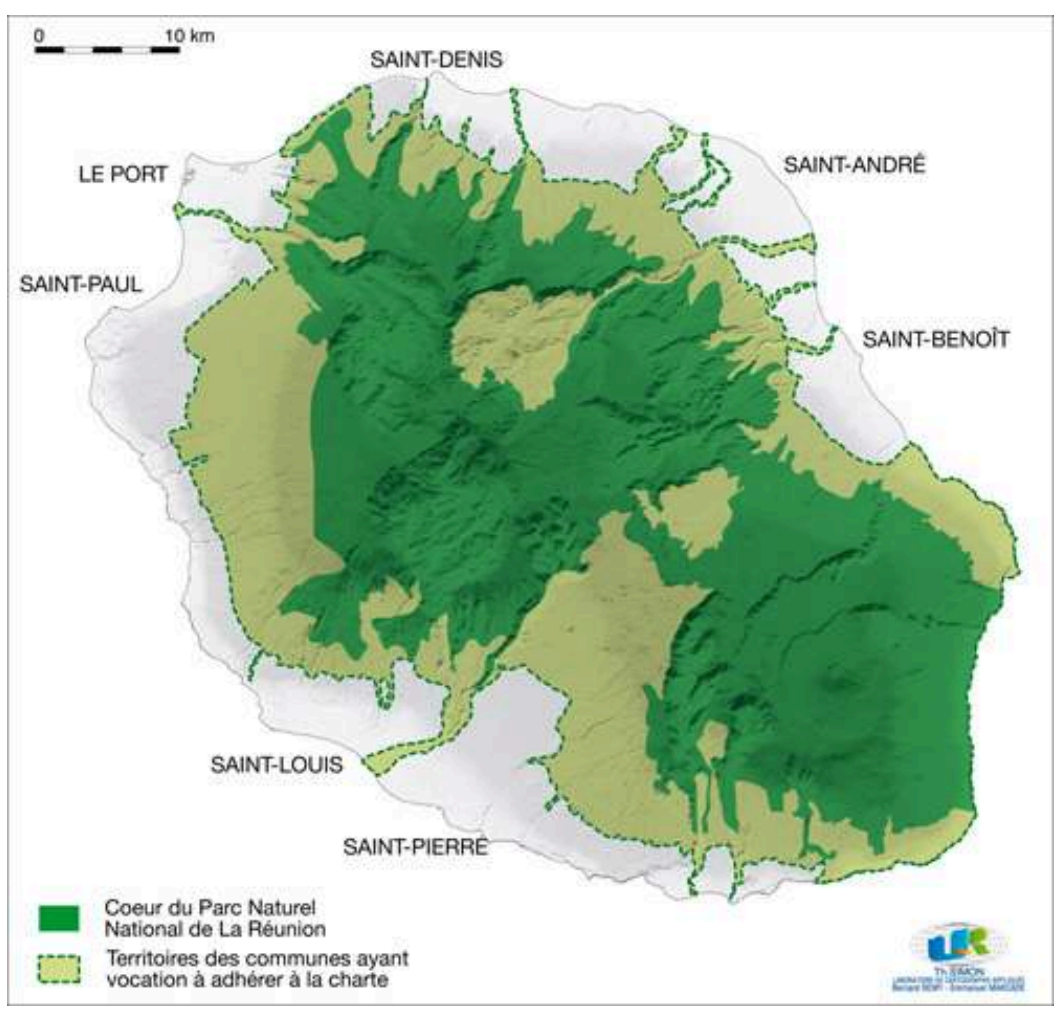

27 La zone « centrale » et la zone " périphérique » (figure 9), dite encore "d'adhésion », constituent bien dans ce projet des territoires de «développement»et non de « conservation ». Le Parc a été présenté et semble bien conçu comme un espace de vie, en forte mutation, un espace de production et d'innovation, de partage et d'accueil. Dans sa phase de mise en oeuvre, le projet qui débute est ainsi l'occasion, pour tous les acteurs «institutionnels» (ONF, DAF, DIREN,...) et de «terrain » (maires, associations,...), de s'entendre et de mettre au point une « charte» : "une charte de développement durable et non pas seulement une charte d'environnement : elle constitue la nouvelle forme du projet commun de territoire, global, à la fois économique, social, culturel et écologique ».(Projet de Parc National des Hauts de La Réunion, 2003).

\section{Conclusion}

La Réunion n'est plus tout à fait cette "île à sucre ", ce territoire insulaire indiaocéanique marqué par un climat, un cadre et un genre de vie, une civilisation créole, perpétuellement englué dans des « problèmes réunionnais ». Elle n'est plus cette île que décrivait encore Defos Du Rau (1960), dans les premières années de la départementalisation. La Réunion demeure très profondément et fort justement attachée au "temps lontan ", à son histoire et à ses difficultés, à ses misères et à ses réussites. Mais, d'une certaine manière, elle a su aussi se projeter en avant et résolument s'engager, en moins d'un demi-siècle de changement et grâce à un soutien budgétaire conséquent de la part de l'Etat, comme de l'Europe, avec un réel dynamisme et une ambition légitime, dans la voie du développement. Des mutations économiques et sociales profondes sont très largement entamées et le territoire insulaire porte, 
partout, les traces de ces mutations: grands programmes d'équipement et grandes infrastructures, développement urbain accéléré, multiplication des pôles d'activité, initiatives multiples et convergentes dans le domaine du développement "durable». Toutefois, ces projets multiples, souvent de grande ampleur, sont en réalité très fragiles, car ils reposent pour l'essentiel sur des transferts financiers massifs dont la " durabilité » peut inquiéter, à juste titre, dans un contexte de contrainte budgétaire et de crise des finances publiques. Des doutes surgissent aussi sur la cohérence de ces projets multiples qui convergent difficilement dans une vision d'ensemble. Elle reste encore largement à construire : la manipulation médiatique qui consiste à transformer de grands projets d'infrastructures routières en opérations exemplaire de « développement durable » ne peut évidemment pas conforter l'émergence d'une solide et sérieuse démarche prospective. À juste titre, La Réunion ambitionne d'être exemplaire dans cette voie du développement «durable » : elle dispose d'atouts, mais sa réussite passe d'abord par une maîtrise réelle et concrète de son territoire, par une plus grande cohérence dans la multitude des projets, dans la construction d'un cadre stratégique qui fasse pleinement consensus et qui puisse constituer une référence dans la durée.

\section{BIBLIOGRAPHIE}

AGORAH, 2001. Aménager La Réunion de 2020 ? Actes du colloque des 22 \& 23 » octobre 2001. Hôtel de Région. Sainte-Clotilde, AGORAH, 83 p.

Conseil Régional, 2006. Pour mieux se déplacer. Avec la Région, La Réunion en chantier. SaintDenis, $32 \mathrm{p}$.

Crusol, J., 2007. Les îles à sucre. De la colonisation à la mondialisation. Bécherel, Les Perséides, $542 \mathrm{p}$.

Defos Du Rau, J., 1960. L'île de La Réunion. Étude de géographie humaine. Bordeaux, Institut de Géographie, Faculté des Lettres, $70 \mathrm{p}$.

Eve, P., 1992. Île à peur. La peur redoutée ou récupérée à La Réunion des origines à nos jours. Saint-André, Océan Editions, 431 p.

IEDOM, 2007. La Réunion en 2006. Saint-Denis, IEDOM, 279 p.

Jauze, J-M., \& Ninon, J., 1999. Dynamiques et expressions de la périurbanisation à La Réunion. Les Cahiers d'Outre-Mer, $\mathrm{N}^{\circ} 206$, p. 143-168.

Jauze, J-M., 2003. Un territoire en cours d'aménagement, in CREGUR \& INSEE, 2003. Atlas de La Réunion. Saint-Denis, INSEE/Université de La Réunion, p. 88-89.

Lajoie, G., 2003. Densités de population, in CREGUR \& INSEE, 2003. Atlas de La Réunion. SaintDenis, INSEE/Université de La Réunion, p. 46-47.

Ninon, J., 1995. La périurbanisation sur l'espace réunionnais. Nice, Faculté des Lettres et Sciences Humaines, $549 \mathrm{p}$. 
Ninon, J., 2003. L'urbanisation, in CREGUR \& INSEE, 2003. Atlas de La Réunion. Saint-Denis, INSEE/Université de La Réunion, p. 90-91.

Parc National de La Réunion, 2001. Premières propositions de principe de zonage, d'aménagement et de gestion. Saint-Denis, $25 \mathrm{p}$.

Parc National de La Réunion, 2003. Principes pour un parc national de nouvelle génration, comité de pilotage du 12 mars 2003. Saint-Denis, $61 \mathrm{p}$.

Rivière, S., 2007. Les ZAC : Un outil d'aménagement de La Réunion. Etat des lieux et analyse. Septembre 2007. Sainte-Clotilde, AGORAH, 26 p.

Simon, Th., 2007. Espaces et paysages littoraux réunionnais : formation et représentations, enjeux et dynamiques. Travaux et documents, n³2, p. 109-130.

\section{NOTES}

1. Pour s'en convaincre pleinement, il suffit par exemple de relire les analyses conduites par Defos Du Rau, (Defos Du Rau, 1960), dans un ouvrage qui, à juste titre, fait encore référence: «L'accroissement démographique annihile en grande partie le résultat de tous les efforts et de tous les progrès économiques. ", p. 629. Plus récemment encore, sur ce thème, (De Palmas, 2005). Entre 2000 et 2006, la population réunionnaise est passée de 717000 à 784000 habitants, avec un accroissement naturel moyen de 1,49\%, (INSEE : http://www.insee.fr/fr/insee_regions/reunion/ zoom/chif_cles/fregdep/fdep974.htm).

2. Comme en témoigne par exemple la phrase introductive à l'intervention du Président du Conseil Régional, en 2001, à l'ouverture du colloque "Aménager La Réunion de 2020 »: « Nous subissons en ce moment, en termes d'aménagement, la "dictature de l'urgence" !", (AGORAH, 2001), p. 4.

3. À noter toutefois que les écoles du cirque de Mafate sont dotées depuis peu de liaisons Internet « haut débit », ...

4. Lettre du Préfet de Région au Président du Conseil Régional, en 2006, ayant pour objet «La révision du SAR - contribution préliminaire de l'Etat » (Préfecture de La Réunion, 15 juin 2006, référence : DDE-prOpet-cARG-06/355).

5. Idem, supra, note 4.

6. En 2006, la croissance économique de La Réunion s'est établie à + 5,05\% « 2006 restera une année de croissance économique soutenue, portée par la consommation des ménages qui a bénéficié d'une accélération de la dynamique des revenus et d'une croissance de l'investissement toujours rapide... ", (IEDOM, 2007), p.10.

7. Par l'autoritaire Gouverneur Vauboulon qui sera destitué rapidement.

8. Par le Gouverneur Delisle.

9. Pour la première tranche (Saint-Paul/Sainte-Marie) : http://www.tramtrain.fr/rubrique.php?id_rubrique=53

10. D'après les informations communiquées après l'accord de Matignon (janvier 2007) :

http://www.regionreunion.com/fr/spip/spip.php?article1430

11. Sur la base des PIB 2006.

12. En matière d'habitat, le dynamisme des mutations est réel: l'habitat insalubre est graduellement résorbé et de très nombreux projets sont engagés : 35 opérations (ZAC) en cours de réalisation, 850 ha en cours d'aménagement dont la moitié dans l'ouest de l'île. Il s'agit d'opérations de taille modeste (surface moyenne de $28 \mathrm{ha}$ ), 18500 logements prévus à terme dans les ZAC de La Réunion (B. Rivière, 2007). 
13. Ainsi, le secteur des BTP est devenu un « moteur » économique essentiel dans l'île : créateur d'entreprises, d'emplois (20000 salariés en 2006) et de richesses (un chiffre d'affaires global dépassant annuellement le milliard d'€ depuis 2005).

\section{RÉSUMÉS}

L'île de La Réunion s'est engagée dans une profonde mutation territoriale. Cette mutation se traduit notamment par de grands travaux d'infrastructures, principalement conduits en vue de faciliter les déplacements, depuis toujours problématiques. Ces chantiers d'ampleur ne doivent toutefois pas occulter, ni les initiatives structurantes de moindre ampleur (par exemple, la multiplication des zones d'activités), ni l'indispensable mise en cohérence et la mise en œuvre effective des encadrements réglementaires multiples dont l'île est dotée en matière d'aménagement du territoire.

The Reunion island is engaged in a deep territorial change. This change results in particular in great work of infrastructures, mainly led in order to facilitate displacements, since always problematic. These works of width should however not occult, neither the structuring initiatives of less width (for example, the multiple zones of activities), nor the essential consistency and the effective setting of the multiple framings lawful whose island is equipped as regards land and regional planning.

\section{INDEX}

Mots-clés : aménagement du territoire, développement durable, île de La Réunion, infrastructures

Keywords : infrastructures, land planning, Reunion island, sustainable development

\section{AUTEUR}

\section{THIERRY SIMON}

Thierry Simon (thierry.simon@univ-reunion.fr) est maître de conférences HDR en géographie à l'Université de La Réunion. Il a récemment publié :- Espaces et paysages littoraux réunionnais : formation et représentation, enjeux et dynamiques. In Jean-Michel Jauze \& Pascal Saffache, (2007). Approches des littoraux réunionnais et martiniquais. Saint-Denis, 2007, Travaux \& Documents, $\mathrm{n}^{\circ} 32$, p.109-130.

- in Jean Michel Jauze (Dir.). L'île Maurice face à ses nouveaux défis. Paris, L'Harmattan, 2008, p. 105-117: Evolution des paysages, avec Karim Jaufeerally, p. 117-137: Des littoraux sous contrainte, p. 205-217: Les mutations agricoles. 